\title{
Oncologist Head and Neck Cancers
}

\section{Intensity-Modulated Radiation Therapy for Head and Neck Carcinoma}

\author{
Vincent Grégoire, ${ }^{a}$ Wilfried De Neve, ${ }^{b}$ Avraham Eisbruch,,${ }^{c}$ Nancy Lee, \\ Danielle Van den Weyngaert, ${ }^{e}$ Dirk VAN Gestel ${ }^{e}$
}

\begin{abstract}
${ }^{a}$ Radiation Oncology Department and Center for Molecular Imaging and Experimental Radiotherapy, Université Catholique de Louvain, St-Luc University Hospital, Brussels, Belgium; ${ }^{b}$ Radiation Oncology Department, Ghent University Hospital, Gent, Belgium; ${ }^{\mathrm{c} D e p a r t m e n t ~ o f ~ R a d i a t i o n ~ O n c o l o g y, ~ U n i v e r s i t y ~ o f ~}$ Michigan, Ann Arbor, Michigan, USA; ${ }^{\mathrm{d} D e p a r t m e n t ~ o f ~ R a d i a t i o n ~ O n c o l o g y, ~ M e m o r i a l ~ S l o a n-K e t t e r i n g ~}$

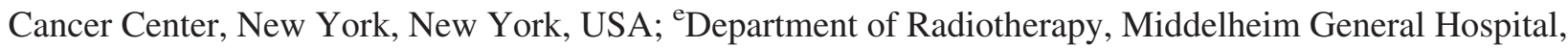
Antwerp, Belgium
\end{abstract}

Key Words. Radiotherapy • Head and neck cancer • IMRT

\section{LEARNING OBJECTIVES}

After completing this course, the reader will be able to:

1. Outline innovations related to targeted radiation therapy.

2. Describe trials proving an advantage using IMRT.

3. Assess treatment planning modalities and how IMRT fields are designed.

4. Evaluate when there is a toxicity advantage for IMRT.

5. Discern when conventional radiotherapy should be used instead of IMRT.

Abstract

Intensity-modulated radiation therapy (IMRT) for head and neck tumors refers to a new approach that aims at increasing the radiation dose gradient between the target tissues and the surrounding normal tissues at risk, thus offering the prospect of increasing the locoregional control probability while decreasing the complication rate. As a prerequisite, IMRT requires a proper selection and delineation of target volumes. For the latter, recent data indicate the potential of functional imaging to complement anatomic imaging modalities. Nonrandomized clinical series in paranasal sinuses and pharyngolaryngeal carcinoma have shown that IMRT was able to achieve a very high rate of locoregional control with less morbidity, such as dry-eye syndrome, xerostomia, and swallowing dysfunction. The promising results of IMRT are likely to be achieved when many treatment conditions are met, for example, optimal selection and delineation of the target volumes and organs at risk, appropriate physical quality control of the irradiation, and accurate patient setup with the use of onboard imaging. Because of the complexity of the various tasks, it is thus likely that these conditions will only be

Correspondence: Vincent Grégoire, M.D., Ph.D., Radiation Oncology Department, St-Luc University Hospital, 10 Avenue Hippocrate, B-1200 Brussels, Belgium. Telephone: 32-2-764-94-43; Fax: 32-2-764-94-25; e-mail: vincent.gregoire@imre.ucl.ac.be Received December 17, 2005; accepted for publication February 22, 2007. CAlphaMed Press 1083-7159/2007/\$30.00/0 doi: 10.1634/theoncologist.12-5-555

The Oncologist 2007;12:555-564 www.TheOncologist.com 
met in institutions having large patient throughput and experience with IMRT. Therefore, patient referral to those institutions is recommended. The Oncologist 2007; 12:555-564

Disclosure of potential conflicts of interest is found at the end of this article.

\section{INTRODUCTION}

During the second half of last century, key technological innovations have tremendously modified the daily practice of radiotherapy, leading to substantial improvements in treatment delivery and outcome. The introduction of linear accelerators (linacs) in the early 1950s, the increasing use of computed tomography (CT) scanning for target volume delineation from the 1980s on, and more recently, in the late 1990s, the availability of advanced treatment planning systems together with multileaf collimators have progressively contributed to a more targeted and conformed dose delivery, that is, a better dose distribution within the target volumes while sparing surrounding normal tissues. In addition, linacs are nowadays equipped with electronic portal imaging devices for verification of patient positioning, thus enabling a better conformity between the planned dose and the dose that is actually delivered. All these technical innovations have led on the one hand to delivering a much higher dose to the target volumes, thus possibly increasing local tumor control, while on the other hand minimizing the undue dose delivered to the surrounding normal tissues, thus possibly decreasing treatment morbidity.

The introduction of inverse treatment planning systems with intensity modulation, that is, intensity-modulated radiation therapy (IMRT), over the last few years has brought another refinement in the ballistics of dose delivery, enabling further improvement in dose delivery and treatment outcome. IMRT is not only a technique for delivering optimized nonuniform beam intensities to a target volume, but it also provides a new approach to the whole treatment procedure from patient immobilization to beam delivery. Implementation of IMRT thus requires a knowledge of setup uncertainties, adequate selection and delineation of target volumes based on optimal imaging modalities, appropriate specification and dose prescription regarding dose-volume constraints, and ad hoc quality control of both the clinical and physical aspects of the whole procedure.

In head and neck (HN) malignancies, IMRT appears to be more and more commonly used for routine treatment, especially in the U.S. [1]. However, only a few randomized trials have demonstrated its superiority over conventional treatments [2] and only a few meaningful retrospective studies are available that demonstrate its potential and its possible drawbacks. The most convincing data on the supe- rior therapeutic gain achievable with IMRT are from tumors close to the base of the skull, such as nasopharyngeal and sinonasal cancers, for which a higher rate of local control and a lower incidence of complications have been reported in comparison with standard two-dimensional (2D) techniques in retrospective comparisons [3,4]. A substantially lower rate of late radiation-induced toxicity, such as xerostomia, has also been extensively documented following the use of IMRT for pharyngolaryngeal squamous cell carcinomas (SCCs) [2, 5-8]. A few retrospective studies have also reported that, despite the high conformality in dose distribution, geographical miss is rather uncommon in IMRT for pharyngolaryngeal tumors, provided that an adequate selection of target volumes is made [9-12].

Although IMRT shows promise as a radiation procedure aimed at increasing therapeutic gain, in the $\mathrm{HN}$ area, it still presents a number of challenges and avenues that have yet to be fully explored. This article presents an overview of the present clinical data supporting the use of IMRT for $\mathrm{HN}$ tumors.

\section{Challenge of Selection and Delineation of THE TARGET VOLUMES}

Among the various steps of the IMRT process, target volume selection and delineation represent, without a doubt, the most dramatic changes in clinical approach compared with the former 2D treatment of HN tumors. As IMRT allows highly conformal dose distribution to target volumes of almost any shape, the adequate selection and delineation of these volumes become of critical importance. An adaptation of the target volumes used in the $2 \mathrm{D}$ approach, in which selection and delineation were determined more by technical limitations than by oncological considerations and patient anatomy, would likely result in no benefit in terms of dose sparing to nontarget tissues. On the other hand, an excessively restrictive selection and delineation of target volumes could easily jeopardize the clinical impact of the highly conformal dose distributions produced.

Over the past few years, several authors have made recommendations for a more precise selection of the clinical target volume (CTV) for both primary tumors and neck nodes $[10,13,14]$. It is beyond the scope of this review article to discuss these recommendations at length. For primary tumors, the issue of selection is briefly discussed in 
the following sections dealing with the various HN sites. For the neck, comprehensive review of clinical, radiological, and pathological data on node distribution for the various sites within the $\mathrm{HN}$ area supports the concept of selective treatment according to nodal stage. In a nutshell, for N0 and N1 patients, selective irradiation of level II-IV or level I-III can be recommended for oropharyngeal, hypopharyngeal, and laryngeal primaries, or for oral cavity primaries, respectively. For nasopharyngeal tumors and for patients with a neck staged as higher than N2a, comprehensive irradiation of all neck levels is recommended.

Guidelines for the delineation of the various node levels in the neck have also been proposed, and a series of consensus recommendations endorsed by major North American (Radiation Therapy Oncology Group [RTOG]) and European (Danish Head and Neck Cancer, European Organization for Research and Treatment of Cancer, Groupe d'Oncologie Radiothérapie Tête et Cou) cooperative groups has been elaborated for the N0 neck $[15,16]$. For the node-positive and the postoperative neck, further recommendations have been recently proposed [17, 18]. It is beyond the scope of this article to discuss these recommendations at length, but the main philosophy of these new additional guidelines was to include extra nodal regions (i.e., retrostyloid area and subclavicular fossa) and/or extra structures (e.g., muscles or gland) found to be at risk for microscopic tumor infiltration.

Another important issue regarding target volume delineation is the choice of the optimal imaging modality used for planning purposes. CT images are typically used because they allow dose calculation with corrections for tissue density inhomogeneity. Contrast medium should be routinely used to allow a much better contrast between normal tissues and tumors, especially because recent data have shown that contrast medium does not substantially influence dose optimization (C. Clark, The Royal Marsden Hospital, London, personal communication). For nasopharyngeal carcinoma, however, magnetic resonance imaging (MRI) has been shown to be superior to CT in reducing interobserver variability $[19,20]$. This advantage of MRI was not observed in oropharyngeal, laryngeal, and hypopharyngeal tumors [21]. A few groups also investigated the role of functional imaging with fluorodeoxyglucosepositron emission tomography (FDG-PET) to delineate gross tumor volume (GTV) [22, 23]. Daisne et al. [22] assessed the accuracy of FDG-PET in comparison with CT for GTV delineation in oropharyngeal, hypopharyngeal, and laryngeal SCC using three-dimensional (3D) registration of the various imaging modalities. In a subset of laryngeal tumors, the imaging modalities could even be compared with the actual surgical specimen taken as a "gold standard." FDG-PET demonstrated higher accuracy in delineating GTV, with a statistically significant smaller target volume. Interestingly, this difference in GTV delineation translated into a difference in CTV and planning target volume (PTV) delineation, which in turn translated into a difference in dose distribution; the dose was much more conformed when FDG-PET was used as the primary imaging modality [24].

Although pretreatment contrast-enhanced CT still presently remains the standard technique used in treatment planning for HNSCC, it is not unlikely that in the near future the introduction of additional modalities may also be considered, not only before treatment, but also possibly during treatment to adapt the GTV to the extent of tumor regression. Furthermore, for PET imaging, tracers other than FDG for targeting biological pathways for radiation response (e.g., proliferation, hypoxia) are under evaluation [25]. The functional assessment of tumor biology may contribute to the delineation of sub-GTV that could benefit from a heterogeneous dose distribution [26]. Although very challenging and extremely promising, these approaches still need thorough validation before they can be introduced into routine clinical practice [27].

\section{ClinicAl EVIDENCE FOR THE USE OF IMRT IN HN TUMORS}

\section{IMRT for Nasopharyngeal Carcinoma}

Because the nasopharynx is situated near numerous critical normal organs, that is, the brain stem and optic chiasm, IMRT is ideal in its attempt to deliver an adequate dose to the gross tumor while sparing these surrounding normal tissues. IMRT plans involving the nasopharynx require contouring the gross disease, which includes the primary gross tumor as well as any gross nodal disease. Subclinical nodal CTV involves bilateral coverage of neck nodal levels delineated up to the base of skull to include the retropharyngeal nodes [4]. For node-negative patients, levels II-V should be contoured. For node-positive cases, inclusion of level IB appears reasonable, although convincing data are missing. In addition to the important at-risk nodal regions, the CTV should also include the entire nasopharynx, clivus, base of skull, pterygoid fossa, parapharyngeal space, inferior sphenoid sinus, and posterior third of the nasal cavity and maxillary sinuses (ensuring adequate margin to the pterygopalatine fossae). The CTV that encompass the above regions can be modified according to tumor (T) stage. A margin around all CTVs should be added to account for patient motion and setup errors, that is, the PTV. This margin is typically $5-6 \mathrm{~mm}$, unless daily imaging and position cor- 
rection are being made, in which case it may be reduced to a minimum of $3 \mathrm{~mm}$ [28].

At Memorial Sloan-Kettering Cancer Center, the implementation of IMRT for nasopharyngeal cases has resulted in significant improvements over the traditional and 3Dconformal radiotherapy (3D-CRT) plans. First, there is better coverage of the retropharynx, base of skull, and medial aspects of the nodal volumes [29]. Xia et al. [30] compared IMRT treatment plans with conventional treatment plans for locally advanced nasopharyngeal cancers. They concluded that IMRT provides better tumor target coverage with significantly better sparing of sensitive normal tissue structures in the treatment of locally advanced nasopharyngeal carcinoma. Another dose distribution study, by Kam et al. [31, 32] from Hong Kong, compared IMRT with 2Dradiotherapy (2D-RT) and 3D-CRT treatment plans. Three patients with different stages, including T1N0, T2bN2, and T4N2, were compared. In all stages, IMRT was noted to have significant dose distribution advantages. In earlystage disease, it provided better parotid gland and temporomandibular joint sparing. In locally advanced disease, it offered better tumor coverage and normal organ sparing and permitted room for dose escalation.

The dose distribution advantages seen with IMRT for nasopharyngeal cancers have also translated into excellent clinical outcomes. At the most recent American Society of Clinical Oncology meeting, Kam et al. [33] presented phase III evidence showing the advantage of IMRT in terms of improvement in xerostomia when compared with conventional radiotherapy. These data add to the data published by Pow et al. [2] demonstrating a significant improvement in quality of life in patients with nasopharyngeal cancer randomized to IMRT compared with those randomized to conventional radiotherapy. In terms of tumor control, the most mature data on local progression-free rates using IMRT for nasopharyngeal cancer come from the University of California at San Francisco (UCSF) (Table 1). With a median follow-up of 31 months, the 4-year local progression-free rate was $97 \%$ while the 4-year regional progression-free rate was $98 \%$ [4]. Bucci et al. [34] have recently updated the UCSF experience and included more patients $(n=118)$. Excellent locoregional progression-free rates were seen. Other important studies have recently emerged out of Hong Kong and are also detailed in Table 1. Although singleinstitution studies show superb outcomes in terms of salivary preservation as well as locoregional control of disease, distant metastasis remains a significant issue in all these series [4, 34-36]. Efforts focused on decreasing the rates of distant metastasis using targeted biologic agents are under way by the RTOG.

An RTOG phase II trial using IMRT with or without chemotherapy for all localized nasopharyngeal cancer has completed accrual and awaits maturity of follow-up data. The protocol is an important one, because it tests whether the ability to achieve excellent control rates in nasopharyngeal cancer patients treated with IMRT can be reproduced in a multi-institutional setting.

The reirradiation of nasopharyngeal cancer has been commonly used and IMRT offers tremendous dose distribution advantages. Reirradiation is more feasible with IMRT than with conventional techniques for the many reasons stated above. Lu et al. [37] have recently reported on their experience with reirradiation using IMRT for recurrent nasopharyngeal cancer. Acute toxicity of the skin, mucosa, and salivary glands was acceptable according to RTOG criteria. Tumor necrosis was seen toward the end of IMRT in 14 patients $(28.6 \%)$. At a median follow-up of 9 months, the locoregional control rate was $100 \%$. Although longer follow-up is necessary, the preliminary toxicity and local control data for these recurrent cases are promising [37].

\section{IMRT for Nasal and Paranasal Sinuses}

By their location, sinonasal tumors are surrounded by critical structures, including the frontal and temporal lobes of the brain, pituitary gland and brainstem, lacrimal glands, eyes, optic nerves, and chiasm. Using conventional radiotherapy techniques, the lacrimal apparatus and the optic pathway structures (retina, optic nerves, chiasm) often received doses equal to the target prescription dose. Conventional radiation therapy for sinonasal cancer resulted in significant ocular toxicity [35, 38-39]. Local control rates of 70\%-90\% in stages T1-T2 and $<50 \%$ in stages T3-T4 were achieved with prescription doses of 56-75 Gy [40]. IMRT allows selective underdosage of organs at risk by creating concave dose distributions around the optic pathway structures together with steep cranial, lateral, and caudal gradients outside the PTV to spare the lacrimal apparatus and the central nervous system. Hypothetically, selective underdosage could decrease toxicity at unchanged target prescription doses.

It is unlikely that answers with level I evidence (evidence generated from randomized clinical trials) will emerge in the foreseeable future because of the rarity of the disease. A PubMed search on November 14, 2005 using IMRT and "paranasal sinus" as key words yielded 23 publications. From these, 17 were on IMRT planning or technical issues. Of the six clinical publications, three reported on $>11$ patients, one from UCSF [41] and two from Ghent University Hospital (GUH) [3, 42].

Using the patient database at GUH, we try to answer the following questions: How do local control and survival 
Table 1. Results from series treating nasopharyngeal cancer with IMRT with or without chemotherapy

\begin{tabular}{|c|c|c|c|c|c|c|c|c|}
\hline Study & $n$ & Staging & $\begin{array}{l}\begin{array}{l}\text { Median } \\
\text { follow-up } \\
\text { (months) }\end{array} \\
\end{array}$ & $\begin{array}{l}\text { Time } \\
\text { point } \\
\text { (years) }\end{array}$ & $\begin{array}{l}\text { Local } \\
\text { control } \\
\text { rate }\end{array}$ & $\begin{array}{l}\text { Regional } \\
\text { control } \\
\text { rate } \\
\end{array}$ & $\begin{array}{l}\text { Distant } \\
\text { metastasis-free } \\
\text { rate }\end{array}$ & $\begin{array}{l}\text { Overall } \\
\text { survival } \\
\text { rate }\end{array}$ \\
\hline Lee et al. [4] (UCSF) & 67 & All stages & 31 & 4 & $97 \%$ & $98 \%$ & $66 \%$ & $73 \%$ \\
\hline Kwong et al. [34] (Hong Kong) & 33 & T1N0-N1, M0 & 24 & 3 & $100 \%$ & $92 \%$ & $100 \%$ & $100 \%$ \\
\hline Kam et al. [31, 32] (Hong Kong) & 64 & All stages & 29 & 3 & $92 \%$ & $98 \%$ & $79 \%$ & $90 \%$ \\
\hline Wolden et al. [36] (MSKCC) & 74 & All stages & 35 & 3 & $91 \%$ & $93 \%$ & $78 \%$ & $83 \%$ \\
\hline
\end{tabular}

Abbreviations: IMRT, intensity-modulated radiation therapy; M, metastasis; MSKCC, Memorial Sloan-Kettering Cancer Center; N, node; T, tumor; UCSF, University of California at San Francisco.

rates of IMRT-treated patients compare with those of historical controls? Can IMRT reduce dry-eye syndrome, visual impairment from retinopathy, and optic neuropathy? Can IMRT reduce nonocular toxicity?

A heterogeneous group (variety in histology, subsite of origin, stage, treatment intent) of 62 patients with sinonasal tumors received IMRT between July 1, 1998 and August 31,2003 . For analysis of survival, local control, and toxicity, we selected patients with stage M0 who underwent R0 resection and postoperative IMRT with curative intent for adenocarcinoma $(n=31)$ or SCC $(n=8)$. The subsite of origin was the ethmoid sinus in 30 patients ( 28 adenocarcinoma), the maxillary sinus in six (all SCC), and the nasal cavity in three patients (all adenocarcinoma). Eleven patients had T4b tumors (2002 International Union Against Cancer Tumor-Node-Metastasis classification) with invasion of the dura or brain through the cribriform plate. Patient characteristics and surgery were described in detail by Duthoy et al. [42].

The 4-year actuarial local control rate after surgery and IMRT was $>80 \%$ for patients with T1-4aN0M0 disease. We compared 28 patients with adenocarcinoma of the ethmoid sinus who received IMRT with a historical control group of 30 patients (all R0-resected ethmoid sinus adenocarcinoma) treated between 1985 and 1994 with 2D $(n=$ 19) techniques, or treated between 1995 and 1998 with 3Dconformal noncoplanar techniques $(n=11)$ at prescription doses of 60-70 Gy (2 Gy/fraction). The 4-year actuarial local control and survival rates for those patients are shown in Table 2.

Fatal relapses occurred within a year after treatment in all patients ( $n=11$ in the IMRT group and $n=3$ in the historical group) with cribriform plate invasion. The present IMRT implementation was clearly not able to reverse the dismal local control rates that are known to exist in stage $\mathrm{T} 4 \mathrm{~b}$ disease with cribriform plate invasion. By excluding patients with cribriform plate invasion, a patient group with good local control and survival could be selected. No con- clusions should be drawn regarding the effect of IMRT on local control as compared with conventional techniques.

In the IMRT group, severe dry-eye syndrome (grade $\geq 3$, persistent pain) was reported in 2 of 39 patients, including a patient with pre-existing unilateral blindness as a result of orbital tumor invasion for which no attempt was made to spare the lacrimal apparatus (Table 3). In the historical control, severe dry-eye syndrome occurred in seven patients, of whom five had been treated by $2 \mathrm{D}$ techniques and two had been treated by 3D techniques (enucleation was required in one patient). A dose-effect analysis in the IMRT group showed that even at doses $<30$ Gy (median dose to the main lacrimal glands) mild forms of dry-eye syndrome could be diagnosed.

Bearing in mind a median follow-up of 32 months in survivors, the data on radiation retinopathy and optic neuropathy should be considered immature. However, we conclude that, during this limited follow-up period, two serious and three mild optic pathway events were recorded. The maximum-dose constraints of $50 \mathrm{~Gy}$ to the retina and $60 \mathrm{~Gy}$ to the optic nerves and chiasm at respective fraction sizes of approximately 1.5 and 1.7 Gy seem to be close to the maximum-tolerated dose for these structures when preservation of vision is the endpoint.

Thus, the 4-year actuarial local control rate after surgery and IMRT was $>80 \%$ for patients with T1-T4aNOM0 disease. Severe dry-eye syndrome could be avoided in almost all patients if attempted. Severe optic pathway injury occurred in about $5 \%$ of patients. We hypothesize that the maximum-tolerated doses to the retina, optic nerves, and chiasm might have been reached in the GUH protocol at 50 Gy, 60 Gy, and 60 Gy in 35 fractions, respectively. This hypothesis is being continuously assessed as more patients are being treated and followed.

\section{IMRT for Oropharyngeal Carcinoma}

Most series reporting clinical results of tumor control rates following IMRT are still quite limited. They are either very 
Table 2. Local control and survival of ethmoid sinus adenocarcinoma

\begin{tabular}{lll}
\hline & $\begin{array}{l}\text { IMRT group } \\
\mathbf{1 9 9 8 - 2 0 0 3}\end{array}$ & $\begin{array}{l}\text { Historical control } \\
\mathbf{1 9 9 8 - 2 0 0 3}\end{array}$ \\
\hline$n$ of patients & 28 & 30 \\
Cribriform plate invasion & 11 & 3 \\
Median follow-up (months) & 31 & 86 \\
Four-year actuarial survival rate & & $66 \%$ \\
$\quad$ All patients & $59 \%$ & $73 \%$ \\
$\quad$ No cribriform plate invasion & $94 \%$ & \\
Four-year actuarial local control rate & $63 \%$ & $63 \%$ \\
$\quad$ All patients & $86 \%$ & $70 \%$ \\
$\quad$ No cribriform plate invasion & \\
\hline Dry-eye symptoms were reported by patients. & \\
Abbreviation: IMRT, intensity-modulated radiation therapy. & .25 \\
\hline
\end{tabular}

heterogeneous regarding tumor sites and stages $[9,11,12$, $41,43]$ or have relatively small patient numbers [8, 41], and all series suffer from a relatively short follow-up. Potential patient selection factors are probably the most important issues that should be considered in assessing outcome in retrospective series of IMRT. IMRT is far more complex and time-consuming than conventional radiotherapy. It is likely that different selection factors play a role at each institution: patients who cannot tolerate lengthy treatment, those judged to be too sick to benefit from complex therapy, those requiring urgent start of therapy, etc., may be selected to receive more simple, conventional treatment. These factors make any attempt to compare the results of different IMRT series, or even series of IMRT with series of conventional RT, futile. Notwithstanding these limitations, all series of IMRT outcome have reported outstanding locoregional control rates for oropharyngeal cancer [11, 12, 43-45]. These series reported 2-year locoregional tumor control rates of $90 \%-98 \%$ for patient populations consisting mainly of stage III-IV tumors. However, these series contain a mixture of definitively and postoperatively treated patients and variable ratios of patients treated with concurrent chemoirradiation or radiotherapy alone. The series from Michigan included patients with various non-nasopharyngeal tumor sites; having an oropharyngeal cancer site was the most significant predictor of local/regional tumor control.

Some of the most reliable information gained from clinical series of IMRT for HN cancer in general and oropharyngeal cancer in particular relates to the pattern of tumor recurrences relative to the targets and the locally delivered doses. These data allow an assessment of the adequacy of target selection and delineation. In all reported cases, it seems that careful selection and delineation of the targets resulted in very few or no marginal or out-of-field recurrences. de Arruda and colleagues reported that all recurrences in their series were in-field [45]. Chao and colleagues reported that most marginal recurrences occurred in the lower neck, which was treated with an anterior field that was matched to the IMRT-treated upper neck [11]. At the University of Michigan, where the majority of patients had oropharyngeal cancer, almost all recurrences occurred in-field, in high-risk volumes that had received the full prescribed doses [9]. An update of this study included 133 patients, 80 of whom had oropharyngeal cancer [12]. At a median follow-up of 32 months 21 locoregional failures $(16 \%)$ occurred. Of these, 17 recurred in-field and four were marginal recurrences. After the first analysis of marginal recurrences [9], modifications in target delineation principles were made, following which no additional marginal recurrences were noted [12]. This demonstrates a learning curve, characteristic of complex therapies, and the likelihood that treatment outcome improves as the experience of the team using IMRT increases. Of note, no marginal recurrence occurred in the contralateral N0 neck, where the cranialmost target included the subdigastric nodes. The definition of the top of level II in the contralateral N0 neck in patients with oropharyngeal cancer has been the level at which the posterior belly of the digastric muscle is crossed by the jugular vein $[9,12,13]$. This definition ensures that the jugulodigastric nodes, being the topmost level II nodes draining oropharyngeal cancer according to Rouviere [46], are irradiated. This definition coincides with the transverse process of the $\mathrm{C} 1$ vertebral body, which marks the top of level II according to consensus guidelines [15]. The lack of recurrences cranial to this level in the Michigan and the Leuven series [9, 47], which used the same definition for the cranialmost level II, is reassuring and is in ac- 
Table 3. Ocular toxicity after radiotherapy for ethmoid tumors

\begin{tabular}{llllll}
\hline \multirow{2}{*}{$\begin{array}{l}\text { Radiation } \\
\text { technique }\end{array}$} & $\boldsymbol{n}$ & Grade 1 & Grade 2 & Dry-eye & Drop in vision \\
\cline { 3 - 5 } & 39 & 2 & 3 & 2 & \multicolumn{2}{c}{ Grade 3 } & 2 \\
IMRT & 19 & $?$ & $?$ & 5 & 0 \\
2D & 11 & $?$ & $?$ & 2 & 2 \\
3D & Abbreviations: 2D, two-dimensional; 3D, three-dimensional; IMRT, intensity-modulated radiation therapy.
\end{tabular}

cordance with the observations of Rouviere [46] and with the consensus recommendations [15]. Two marginal recurrences in the Michigan series were noted in the lateral retropharyngeal nodes (both occurred in patients with oropharyngeal cancer), in which the cranialmost extent of the retropharyngeal nodal targets was defined at the top of C1, according to the observations of Rouviere [46] of the locations of the lateral retropharyngeal nodes. Following these observations, we currently define the retropharyngeal nodes through the base of the skull. Interesting observations about marginal or out-of-field recurrences were reported in the series from the University of Iowa [43]. They found several cases of recurrence in the contralateral level I in patients with lateral oral cavity and oropharyngeal cancer, underscoring the need to include these nodes in locoregionally advanced oral cancer. As more data about marginal recurrences are accumulated, a better understanding and higher certainty are expected to be gained in defining the targets, which will lead to better tumor control and to an ability to further spare noninvolved tissues.

\section{IMRT for Laryngeal Carcinoma}

Comparative dose distribution studies have shown that IMRT can improve the target dose homogeneity in laryngeal and hypopharyngeal SCC while reducing the dose to normal tissues at risk [48-50]. Clinical data on IMRT for laryngeal and hypopharyngeal SCC are, however, quite scarce and typically include patients with definitive and postoperative irradiation with or without induction or concomitant chemotherapy. Chao et al. [11] and Dawson et al. [9] reported very limited series with $<10$ patients in each location, from which definitive conclusions cannot be drawn. Yao et al. [43] reported on a series of 33 patients with laryngeal SCC; the 2-year locoregional control rate reached $85 \%$, which was significantly lower than that observed in oropharyngeal tumors [43]. Patient selection bias cannot, however, be ruled out. A recent study reported the feasibility of a dose-escalation trial in laryngeal SCC using IMRT [51].

\section{LESS NORMAL TISSUE MORBIDITY WITH IMRT}

Several clinical studies assessed the utility of IMRT in parotid salivary gland sparing and in reducing xerostomia. At the University of Michigan, the partial parotid gland doses and volumes following multisegmental IMRT were correlated with selective salivary output from each parotid gland [5]. It was found that the output related to the mean doses to the glands. The large majority of the glands that received a mean dose $>26$ Gy did not produce measurable saliva and did not recover, whereas glands that received lower mean doses produced variable salivary output that increased over time. One year after radiotherapy, parotid glands that received a moderate dose (mean dose, 17-26 Gy) recovered, on average, to the pretreatment salivary production levels [6]. When the doses to the parotid glands were very low, as in cases of unilateral neck radiotherapy in which the contralateral glands received mean doses $<10 \mathrm{~Gy}$, an "overcompensation" of the damage to the ipsilateral glands was noted in the second year post-therapy. In particular, the salivary flow rates from the contralateral glands exceeded, on average, their pretreatment flow rates. This finding motivates reducing the doses to the salivary glands to as low levels as possible.

Data on dose response in the parotid glands are accumulating $[10,52-55]$. The common finding with all these data is that a relationship seems to exist between the mean doses to the glands and their residual salivary output. It is apparent from the studies presented in the review that very different mean doses have been reported as thresholds beyond which functional deficit occurs. These doses are in the range of $20 \mathrm{~Gy}$ to almost $40 \mathrm{~Gy}$. What is the reason for this discrepancy? Several explanations are possible. One explanation relates to different methodologies in assessing salivary flow. Some studies used selective parotid outflow measurements, others used whole mouth saliva, assuming no contribution from the submandibular glands (which had received high doses) and no contribution from the minor salivary glands (whose output is relatively low), and some studies used scintigraphy techniques to assess parotid gland 
function. Beyond this confounding factor, there are clinical factors, beyond radiation dose, that affect the salivary output but have not been taken into account in most of these studies. These factors include dehydration, common in patients receiving $\mathrm{HN}$ radiotherapy, and various medicines found to significantly affect salivary flow rates $[5,6]$. An additional important factor is one reported recently by investigators from the University Hospital in Groningen, The Netherlands [56]. They irradiated different parts of rat salivary glands using high-precision proton radiation and found regional differences in dose-salivary production relationships. Such regional differences are likely to exist in the human parotid glands, and they may be the reason for different results found by researchers using different radiotherapy techniques, which produce different dose distributions within the glands. Further research into intraparotid regional differences in sensitivity to radiation is required for a better understanding of radiation limits. Regardless of the dose threshold, it is now apparent that spared glands not only partly retain the salivary output, but the output increases over time through at least 2 years after radiotherapy [6], compared with generally no improvement over time following standard radiotherapy, in which most of the parotid glands receive full radiotherapy doses.

Once a reasonable preservation of the salivary output is achieved, can we expect similar improvements in patientreported xerostomia symptoms? The correlation between salivary output from the major salivary glands and xerostomia symptoms is significant, but is not very high. Part of the reason for this less-than-straightforward relationship lies in the contribution of the minor salivary glands. These glands are scattered throughout the oral cavity, notably on the surface of the palate, and produce much of the salivary mucin. It has been found that the dose delivered to the oral cavity, serving as a surrogate for the residual function of the minor salivary glands (whose output cannot be measured), was an independent factor in patient-reported xerostomia after radiotherapy, similar to the importance of doses to the major salivary glands [6]. An optimal practice is to add sparing of the oral cavity as an objective in radiotherapy planning, in addition to sparing of the parotid glands. An analysis at the University of Michigan of a validated patient-reported xerostomia questionnaire demonstrated that xerostomia improved significantly over time, in tandem with an increase in saliva production [6]. Two years following irradiation, xerostomia reported by patients receiving parotid-sparing bilateral neck radiation was only slightly worse than that in patients receiving unilateral neck radiotherapy. Investigators at UCSF also reported an improvement to mild or no xerostomia during the second year after IMRT for nasopharyngeal cancer $[41,57]$. It is apparent from all of these stud- ies that the partial sparing of the salivary glands, made possible by IMRT, achieves tangible gains both in the retention of salivary production and in the symptoms of xerostomia.

Another issue in the efforts to reduce xerostomia by IMRT is the importance of the submandibular glands. These glands lie anterior to the level II lymph node targets in the neck. It is not possible to spare a substantial amount of these glands while treating both sides of the neck (which is required for all advanced $\mathrm{HN}$ cancer), resulting in no measurable salivary output from these glands after radiation [6]. Whether the use of technology like proton beams would help is not yet known. Canadian investigators who moved one submandibular gland to the submental space, away from the radiotherapy fields, reported an impressive reduction in xerostomia [58]. However, this technique has not yet gained broad acceptance, as submandibular lymph nodes are so closely related to the gland, or even into the gland, that transposition may not only spare the organ at risk but also the target tissue.

Additional potential functional gains from IMRT compared with conventional radiotherapy include swallowing and speech measures, reported to be superior using IMRT compared with standard radiotherapy [59]. These potential benefits may translate into improvements in broad aspects of quality of life [60]. Thus, IMRT of HN cancer may achieve broad improvements in quality of life rather than be limited to improvements in xerostomia alone. Efforts to identify the structures whose damage causes long-term dysphagia and aspiration, and to determine IMRT strategies that may spare these structures without underdosing the targets, have been reported [61]. An assessment of the clinical effect of these efforts is ongoing. In a recent study, however, a dosimetric comparison showed a much higher dose to the larynx when the entire neck was irradiated with IMRT than with a classical three-field setup with an anterior field shielding the midline structures [62]. To avoid such a perverse effect of IMRT, the laryngeal and hypopharyngeal structures have to be delineated as organs at risk, and assigned with a dose-volume constraint.

\section{CONCLUSION}

The data accumulated so far tend to indicate that this new technique increases the therapeutic ratio by decreasing treatment morbidity such as xerostomia. Whether all patients with HNSCC will benefit from IMRT is still unknown. We hypothesize that patients with very early disease (e.g., stage I tumors) or very extended disease (e.g., stage IVb tumors) will have less benefit from this technique, the former because a very high therapeutic ratio is 
already achieved and the latter because extended target volumes will need to be irradiated.

The promising results of IMRT can, however, be achieved only when all treatment conditions are met, for example, optimal selection and delineation of the target volumes and organs at risk, appropriate physical quality control of the irradiation, and accurate patient setup with the use of onboard imaging or more advanced imaging like CT during therapy [28]. Because of the complexity of the various tasks, it is thus likely that these conditions will be met only in institutions having a large patient throughput. Therefore, patient referral to those institutions with experience in treating many patients with IMRT of HN cancer is recommended.

\section{Disclosure of Potential Conflicts OF INTEREST}

The authors indicate no potential conflicts of interest.

\section{REFERENCES}

1 Mell LK, Roeske JC, Mundt AJ. A survey of intensity-modulated radiation therapy use in the United States. Cancer 2003;98:204-211.

2 Pow EH, Kwong DL, McMillan AS et al. Xerostomia and quality of life after intensity-modulated radiotherapy vs. conventional radiotherapy for early-stage nasopharyngeal carcinoma: Initial report on a randomized controlled clinical trial. Int J Radiat Oncol Biol Phys 2006;66:981-991.

3 Claus F, Boterberg T, Ost P et al. Short term toxicity profile for 32 sinonasal cancer patients treated with IMRT. Can we avoid dry eye syndrome? Radiother Oncol 2002;64:205-208.

4 Lee N, Xia P, Quivey JM et al. Intensity-modulated radiotherapy in the treatment of nasopharyngeal carcinoma: An update of the UCSF experience. Int J Radiat Oncol Biol Phys 2002;53:12-22.

5 Eisbruch A, Ten Haken RK, Kim HM et al. Dose, volume, and function relationships in parotid salivary glands following conformal and intensitymodulated irradiation of head and neck cancer. Int J Radiat Oncol Biol Phys 1999;45:577-587.

6 Eisbruch A, Kim HM, Terrell JE et al. Xerostomia and its predictors following parotid-sparing irradiation of head-and-neck cancer. Int J Radiat Oncol Biol Phys 2001;50:695-704.

7 Chao KS, Majhail N, Huang CJ et al. Intensity-modulated radiation therapy reduces late salivary toxicity without compromising tumor control in patients with oropharyngeal carcinoma: A comparison with conventional techniques. Radiother Oncol 2001;61:275-280.

8 Henson BS, Inglehart MR, Eisbruch A et al. Preserved salivary output and xerostomia-related quality of life in head and neck cancer patients receiving parotid-sparing radiotherapy. Oral Oncol 2001;37:84-93.

9 Dawson LA, Anzai Y, Marsh L et al. Patterns of local-regional recurrence following parotid-sparing conformal and segmental intensity-modulated radiotherapy for head and neck cancer. Int J Radiat Oncol Biol Phys 2000; 46:1117-1126.

10 Chao KS, Wippold FJ, Ozyigit G et al. Determination and delineation of nodal target volumes for head-and-neck cancer based on patterns of failure in patients receiving definitive and postoperative IMRT. Int J Radiat Oncol Biol Phys 2002;53:1174-1184.

11 Chao KS, Ozyigit G, Tran BN et al. Patterns of failure in patients receiving definitive and postoperative IMRT for head-and-neck cancer. Int J Radiat Oncol Biol Phys 2003;55:312-321.

12 Eisbruch A, Marsh LH, Dawson LA et al. Recurrences near base of skull after IMRT for head-and-neck cancer: Implications for target delineation in high neck and for parotid gland sparing. Int J Radiat Oncol Biol Phys 2004; 59:28-42.

13 Eisbruch A, Foote RL, O'Sullivan B et al. Intensity-modulated radiation therapy for head and neck cancer: Emphasis on the selection and delineation of the targets. Semin Radiat Oncol 2002;12:238-249.
14 Grégoire V, Coche E, Cosnard G et al. Selection and delineation of lymph node target volumes in head and neck conformal radiotherapy. Proposal for standardizing terminology and procedure based on the surgical experience. Radiother Oncol 2000;56:135-150.

15 Grégoire V, Levendag P, Ang KK et al. CT-based delineation of lymph node levels and related CTVs in the node negative neck: DAHANCA, EORTC, GORTEC, RTOG consensus guidelines. Radiother Oncol 2003; 69:227-236.

16 Levendag P, Braaksma M, Coche E et al. Rotterdam and Brussels CT-based neck nodal delineation compared with the surgical levels as defined by the American Academy of Otolaryngology-Head and Neck Surgery. Int J Radiat Oncol Biol Phys 2004;58:113-123.

17 Apisarnthanarax S, Elliott DD, El-Naggar AK, et al. Determining optimal clinical target volume margins in head-and-neck cancer based on microscopic extracapsular extension of metastatic neck nodes. Int J Radiat Oncol Biol Phys 2006;64:678-683.

18 Grégoire V, Eisbruch A, Hamoir M et al. Proposal for the delineation of the nodal CTV in the node-positive and the post-operative neck. Radiother Oncol 2006;79:15-20.

19 Rasch C, Keus R, Pameijer FA et al. The potential impact of CT-MRI matching on tumor volume delineation in advanced head and neck cancer. Int J Radiat Oncol Biol Phys 1997;39:841-848.

20 Steenbakkers R, Duppen J, Fitton I et al. Observer variation in delineation of nasopharyngeal carcinoma for radiotherapy, A 3-D analysis. Int J Radiat Oncol Biol Phys 2004;60(suppl):S160-S161.

21 Geets X, Daisne JF, Arcangeli S et al. Inter-observer variability in the delineation of pharyngo-laryngeal tumor, parotid glands and cervical spinal cord: Comparison between CT-scan and MRI. Radiother Oncol 2005;77: $25-31$.

22 Daisne JF, Duprez T, Weynand B et al. Tumor volume in pharyngolaryngeal squamous cell carcinoma: Comparison at CT, MR imaging, and FDG PET and validation with surgical specimen. Radiology 2004;233:93-100.

23 Paulino AC, Koshy M, Howell R et al. Comparison of CT- and FDG-PETdefined gross tumor volume in intensity-modulated radiotherapy for headand-neck cancer. Int J Radiat Oncol Biol Phys 2005;61:1385-1392.

24 Geets X, Daisne JF, Tomsej M, et al. Impact of the type of imaging modality on target volumes delineation and dose distribution in pharyngo-laryngeal squamous cell carcinoma: Comparison between pre- and per-treatment studies. Radiother Oncol 2006;78:291-297.

25 Chao KS, Bosch WR, Mutic S et al. A novel approach to overcome hypoxic tumor resistance: $\mathrm{Cu}$-ATSM-guided intensity-modulated radiation therapy. Int J Radiat Oncol Biol Phys 2001;49:1171-1182.

26 Ling CC, Humm J, Larson S et al. Towards multidimensional radiotherapy (MD-CRT): Biological imaging and biological conformality. Int J Radiat Oncol Biol Phys 2000;47:551-560. 
27 Frank SJ, Chao KS, Schwartz DL et al. Technology insight: PET and $\mathrm{PET} / \mathrm{CT}$ in head and neck tumor staging and radiation therapy planning. Nat Clin Pract Oncol 2005;2:526-533.

28 Feng M, Eisbruch A. Future issues in highly conformal radiotherapy for head and neck cancer. J Clin Oncol 2007;25:1009-1013.

29 Hunt MA, Hsiung CY, Spirou SV et al. Evaluation of concave dose distributions created using an inverse planning system. Int J Radiat Oncol Biol Phys 2002;54:953-962.

30 Xia P, Lee N, Liu YM et al. A study of planning dose constraints for treatment of nasopharyngeal carcinoma using a commercial inverse treatment planning system. Int J Radiat Oncol Biol Phys 2004;59:886-896.

31 Kam MK, Chau RM, Suen J et al. Intensity-modulated radiotherapy in nasopharyngeal carcinoma: Dosimetric advantage over conventional plans and feasibility of dose escalation. Int J Radiat Oncol Biol Phys 2003;56: $145-157$.

32 Kam MK, Teo PM, Chau RM et al. Treatment of nasopharyngeal carcinoma with intensity-modulated radiotherapy: The Hong Kong experience. Int J Radiat Oncol Biol Phys 2004;60:1440-1450.

33 Kam MK, Leung SF, Zee B et al. Impact of intensity-modulated radiotherapy (IMRT) on salivary gland function in early-stage nasopharyngeal carcinoma (NPC) patients: A prospective randomized study. J Clin Oncol 2005;23(suppl 1):500S.

34 Bucci M, Xia P, Lee N et al. Intensity modulated radiation therapy for carcinoma of the nasopharynx: An update of the UCSF experience. Int J Radiat Oncol Biol Phys 2004;60:S317-S318.

35 Kwong DL, Pow EH, Sham JS et al. Intensity-modulated radiotherapy for early-stage nasopharyngeal carcinoma: A prospective study on disease control and preservation of salivary function. Cancer 2004;101:1584-1593.

36 Wolden SL, Chen WC, Pfister DG et al. Intensity-modulated radiation therapy (IMRT) for nasopharynx cancer: Update of the Memorial SloanKettering experience. Int J Radiat Oncol Biol Phys 2006;64:57-62.

37 Lu TX, Mai WY, Teh BS et al. Initial experience using intensity-modulated radiotherapy for recurrent nasopharyngeal carcinoma. Int J Radiat Oncol Biol Phys 2004;58:682-687.

38 Parsons JT, Bova FJ, Fitzgerald CR et al. Severe dry-eye syndrome following external beam irradiation. Int J Radiat Oncol Biol Phys 1994;30:775780

39 Parsons JT, Bova FJ, Mendenhall WM et al. Response of the normal eye to high dose radiotherapy. Oncology (Williston Park) 1996;10:837-847; discussion $847-848,851,852$.

40 Dulguerov P, Jacobsen MS, Allal AS et al. Nasal and paranasal sinus carcinoma: Are we making progress? A series of 220 patients and a systematic review. Cancer 2001;92:3012-3029.

41 Lee N, Xia P, Fischbein NJ et al. Intensity-modulated radiation therapy for head-and-neck cancer: The UCSF experience focusing on target volume delineation. Int J Radiat Oncol Biol Phys 2003;57:49-60.

42 Duthoy W, Boterberg T, Claus F et al. Postoperative intensity-modulated radiotherapy in sinonasal carcinoma: Clinical results in 39 patients. Cancer 2005;104:71-82.

43 Yao M, Dornfeld KJ, Buatti JM et al. Intensity-modulated radiation treatment for head-and-neck squamous cell carcinoma-the University of Iowa experience. Int J Radiat Oncol Biol Phys 2005;63:410-421.

44 Butler EB, Teh BS, Grant WH 3rd et al. SMART (simultaneous modulated accelerated radiation therapy) boost: A new accelerated fractionation schedule for the treatment of head and neck cancer with intensity modulated radiotherapy. Int J Radiat Oncol Biol Phys 1999;45:21-32.

45 de Arruda FF, Puri DR, Zhung J et al. Intensity-modulated radiation therapy for the treatment of oropharyngeal carcinoma: The Memorial Sloan-Kettering Cancer Center experience. Int J Radiat Oncol Biol Phys 2006;64: 363-373.

46 Rouviere H. Lymphatic Systems of the Head and Neck. Ann Arbor, MI: Edwards Brothers, 1938.

47 Bussels B, Maes A, Hermans R et al. Recurrences after conformal parotidsparing radiotherapy for head and neck cancer. Radiother Oncol 2004;72: $119-127$.

48 Braaksma MM, Wijers OB, Levendag PC et al. Optimisation of conformal radiation therapy by intensity modulation: Cancer of the larynx and salivary gland function. Radiother Oncol 2003;66:291-302.

49 Clark $\mathrm{CH}$, Bidmead AM, Nutting $\mathrm{CM}$ et al. Intensity-modulated radiotherapy improves target coverage, spinal cord sparing and allows dose escalation in patients with locally advanced cancer of the larynx. Radiother Oncol 2004;70:189-198.

50 Penagaricano JA, Ratanatharathorn V, Papanikolaou $\mathrm{N}$ et al. Intensitymodulated radiation therapy reduces the dose to normal tissue in T2N0M0 squamous cell carcinoma of the glottic larynx. Med Dosim 2004;29:254257.

51 Guerrero Urbano MT, Clarck C, Nutting C et al. Results of intensity modulated radiotherapy (IMRT) in laryngeal and hypopharyngeal cancer: A dose escalation study. Eur J Cancer Suppl 2005;3:287.

52 Roesink JM, Konings AW, Terhaard $\mathrm{CH}$ et al. Preservation of the rat parotid gland function after radiation by prophylactic pilocarpine treatment: Radiation dose dependency and compensatory mechanisms. Int J Radiat Oncol Biol Phys 1999;45:483-489.

53 Maes A, Weltens C, Flamen P et al. Preservation of parotid function with uncomplicated conformal radiotherapy. Radiother Oncol 2002;63:203211.

54 Bussels B, Maes A, Flamen P et al. Dose-response relationships within the parotid gland after radiotherapy for head and neck cancer. Radiother Oncol 2004;73:297-306.

55 Munter MW, Karger CP, Hoffner SG et al. J. Evaluation of salivary gland function after treatment of head-and-neck tumors with intensity-modulated radiotherapy by quantitative pertechnetate scintigraphy. Int J Radiat Oncol Biol Phys 2004;58:175-184.

56 Konings AW, Coppes RP, Vissink A. On the mechanism of salivary gland radiosensitivity. Int J Radiat Oncol Biol Phys 2005;62:1187-1194.

57 Sultanem K, Shu HK, Xia P et al. Three-dimensional intensity-modulated radiotherapy in the treatment of nasopharyngeal carcinoma: The University of California-San Francisco experience. Int J Radiat Oncol Biol Phys 2000; 48:711-722.

$58 \mathrm{~J}$ a N, Seikaly H, Harris J et al. Prevention of radiation induced xerostomia by surgical transfer of submandibular salivary gland into the submental space. Radiother Oncol 2003;66:283-289.

59 Mittal BB, Pauloski BR, Haraf DJ et al. Swallowing dysfunction-preventative and rehabilitation strategies in patients with head-and-neck cancers treated with surgery, radiotherapy, and chemotherapy: A critical review. Int J Radiat Oncol Biol Phys 2003;57:1219-1230.

60 Lin A, Kim HM, Terrell JE et al. Quality of life following parotid-sparing IMRT of head and neck cancer: A prospective longitudinal study. Int J Radiat Oncol Biol Phys 2003;57:61-70.

61 Eisbruch A, Schwartz M, Rasch C et al. Dysphagia and aspiration after chemoradiotherapy for head-and-neck cancer: Which anatomic structures are affected and can they be spared by IMRT? Int J Radiat Oncol Biol Phys 2004;60:1425-1439.

62 Amdur RJ, Li JG, Liu C et al. Unnecessary irradiation in the IMRT era. Head Neck 2004;26:257-263; discussion 263-264. 